\title{
Metalloenzymes: Use of Recombinant Protein Expression and Affinity Tags to Aid Identification of Native Metal Ion Cofactors
}

\section{Evren Kocabas and Marcy Hernick*}

Department of Biochemistry, Virginia Tech, Blacksburg, VA 24061, USA

Metalloenzymes remain an active area of research due in part to successes in targeting these enzymes for drug development. An estimated $10 \%$ of approved drugs target metalloenzymes [1], including angiotensin-converting enzyme, carbonic anhydrase, and matrix metalloprotesases [2-5]. Since inhibitors of metalloenzymes often contain a metal-binding group that targets the catalytic metal ion, the interaction between the metal-binding group and catalytic metal ion will significantly impact inhibitor affinity for the target enzyme. Therefore, the development of therapeutically effective inhibitors requires proper identification of the biologically relevant metalloenzyme cofactor(s).

Our recent editorial highlighted the increasing number of zinc proteins that have been reclassified as iron or cambialistic proteins, the importance of metal environment in cofactor selection, how traditional methods for protein expression and purification are inherently biased towards incorporation of zinc into metalloenzymes, and described steps that can be taken to minimize experimental artifacts [6]. Since multiple metal ions are often capable of serving as efficient cofactors for a given metalloenzyme in vitro, identification of the native cofactor is largely based on the identity of the metal ion that co-purifies with the protein of interest. Herein we compare different strategies of recombinant protein expression and purification used to aid in the identification of native cofactors with the following enzymes: histone deacetylase 8 (HDAC8), UDP-3-O-(R-3-hydroxymyristoyl)- $N$-acetylglucosamine deacetylase (LpxC), S-Ribosylhomocysteinase (LuxS), N-acetyl-1D-myo-inosityl-2-amino-2-deoxy- $\alpha$-D glucopyranoside deacetylase (MshB), and peptide deformylase (PDF).

Ideally experiments aimed at identification of the native cofactor would rely on isolation of the native enzyme from the native source without the use of recombinant protein expression or affinity tags. However, this is not always possible due to the instability of enzymes, low levels of expression of native proteins, and the lengthy purification procedures required (i.e., multiple columns, precipitation steps) that allow for metal dissociation and/or exchange during the purification process. For example, initial attempts to isolate native PDF and LuxS from E. coli were unsuccessful due to the instability of these proteins [7-11]. Years later, recombinant EcPDF overexpressed in E. coli (LB media supplemented with $\mathrm{Zn}^{2+}$ ) was purified using a combination of ammonium sulfate fractionation, gel filtration, anion exchange, and hydrophobic interaction chromatography [9]. Isolated EcPDF contained bound $\mathrm{Zn}$ (0.1-1.2 $\mathrm{Zn}^{2+} /$ protein) and irreproducible amounts of iron, copper, and cobalt $\left(\leq 0.3 \mathrm{Me}^{2+} /\right.$ protein) [9]. Further studies revealed isolated EcPDF to be a mixture of $\mathrm{Zn}^{2+}$ - and $\mathrm{Fe}^{2+}$-PDF, with $\mathrm{Fe}^{2+}$-PDF having the higher activity [12]. Based on the biochemical properties of $\mathrm{Zn}^{2+}$ - and $\mathrm{Fe}^{2+}-\mathrm{PDF}$, native PDF was identified as a $\mathrm{Fe}^{2+}$ enzyme. The instability of native PDF is attributed to the redox sensitivity of the $\mathrm{Fe}^{2+}$ cofactor and oxidation of $\mathrm{Fe}^{2+}$ to the catalytically inactive $\mathrm{Fe}^{3+}$ [13]. In contrast to EcPDF, native PDF from the Fe-limited Borrelia burgdorferi contains $\mathrm{Zn}^{2+}$ and is stable under aerobic conditions [14]. These studies on PDF show how recombinant protein expression has advanced the field of metalloenzyme research by allowing for isolation of sufficient quantities of proteins that can be characterized to identify biochemical properties, which can aid identification of the native cofactor. However, constructs lacking affinity tags require lengthy purification procedures that allow for metal ion dissociation and/or exchange, and therefore are not ideal for probing enzymes with oxygen sensitive cofactors (i.e., $\left.\mathrm{Fe}^{2+}\right)$.

The field of metalloenzyme research has since moved to using recombinant protein expression with affinity tags. The affinity tags enable rapid isolation of the target protein in one to two steps, thereby circumventing problems associated with protein instability and minimizing metal dissociation/exchange during the purification process. The most commonly used affinity tag to date is the His-tag. Expression of His-BbPDF in minimal media supplemented with specific metal ions $\left(\mathrm{ZnCl}_{2}, \mathrm{CoCl}_{2}\right.$ or $\left.\mathrm{FeCl}_{3}\right)$ enabled preparation of $\mathrm{Zn}^{2+}, \mathrm{Co}^{2+}$, and $\mathrm{Fe}^{2+}$ PDF following Co-IMAC purification [14]. The biochemical properties of these enzymes were compared to those of native BbPDF, which aided identification of $\mathrm{Zn}^{2+}$ as the native cofactor. The ratio of $\mathrm{Fe}^{2+} / \mathrm{PDF}$ obtained in these studies was less than 1, highlighting that a loss of $\mathrm{Fe}^{2+}$ from proteins occurs with aerobic purification procedures. Similarly, His-LuxS constructs were used to help identify LuxS from E. coli and Bacillus subtilis as $\mathrm{Fe}^{2+}$-dependent enzymes [10]. His-LuxS expressed in E. coli (LB media) and purified by Co-IMAC contains mixture of bound $\mathrm{Fe}$ (0.45), $\mathrm{Co}(0.15)$ and $\mathrm{Zn}(0.33)$, while His-LuxS expressed in minimal media supplemented with specific metals produced the $\mathrm{Zn}^{2+}$ , $\mathrm{Co}^{2+}$, and $\mathrm{Fe}^{2+}$-His-LuxS proteins following Co-IMAC purification, which allowed for biochemical characterization of these proteins. Partially purified native LuxS exhibited similar biochemical properties to $\mathrm{Fe}^{2+}$-LuxS (i.e., air instability, $\mathrm{H}_{2} \mathrm{O}_{2}$ sensitivity) leading to the identification of $\mathrm{Fe}^{2+}$ as the native cofactor for LuxS. Similar to EcPDF, the instability of native LuxS is due to the redox sensitivity of the $\mathrm{Fe}^{2+}$ cofactor. The His-tag was also used for the purification of HDAC8 [15]. In contrast to the above examples, these studies used a cleavable His-tag construct to enable isolation of the untagged target protein following removal of the His-tag upon treatment with TEV protease and a second IMAC column (to remove His-TEV and uncleaved His-HDAC8). This approach ensures that the detected metal ions are bound to the protein of interest and not the His-tag. His-HDAC8 expressed in E. coli (LB media supplemented with $\mathrm{Zn}^{2+}$ ) and purified using Ni-IMAC contained bound $\mathrm{Fe}(0.8), \mathrm{Ni}(0.5)$, and $\mathrm{Zn}(0.1)$. Following TEV cleavage of the His-tag, dialysis, and the second Ni-IMAC column, purified HDAC8 contained bound $\mathrm{Fe}$ (0.03), $\mathrm{Ni}(0.2)$, and $\mathrm{Zn}(1.0)$. Sufficient quantities

*Corresponding author: Marcy Hernick, Department of Biochemistry, Virginia Tech, Blacksburg, VA 24061, USA, Tel: 540-231-2842; Fax: 540-231-9070; E-mail: hernickm@vt.edu

Received February 05, 2013; Accepted February 06, 2013; Published February 08, 2013

Citation: Kocabas E, Hernick M (2013) Metalloenzymes: Use of Recombinant Protein Expression and Affinity Tags to Aid Identification of Native Metal Ion Cofactors. Biochem Anal Biochem 2:132. doi:10.4172/2161-1009.1000132

Copyright: ( $) 2013$ Kocabas E, et al. This is an open-access article distributed under the terms of the Creative Commons Attribution License, which permits unrestricted use, distribution, and reproduction in any medium, provided the original author and source are credited. 
of isolated protein also allowed for the biochemical characterization of $\mathrm{Zn}^{2+}-, \mathrm{Co}^{2+}$, and $\mathrm{Fe}^{2+}$-HDAC8 for comparison to the native enzyme activity. The activity of crude lysates containing HDAC8 are higher when the growth media is supplemented with iron and is aerobically sensitive suggesting that most of the HDAC8 is present as $\mathrm{Fe}^{2+}-\mathrm{HDAC} 8$ when expressed in E. coli. The loss of Fe during the purification process is attributed to oxidation of $\mathrm{Fe}^{2+}$ to $\mathrm{Fe}^{3+}$, dissociation of $\mathrm{Fe}^{3+}$ from HDAC8, and replacement with $\mathrm{Zn}^{2+}$ (from dialysis buffers). The bound $\mathrm{Ni}$ is attributed to the Ni-IMAC purification. A TEV cleavable His-tag was also used with MshB, and results from these experiments showed that the identity of the metal ion bound to purified MshB largely reflected the metal ion used in the IMAC purification $(\mathrm{Ni}, \mathrm{Co}, \mathrm{Zn}$, or Fe) [16]. These examples demonstrate the usefulness of recombinant protein expression in the identification of native cofactors. While the His-tag allows for purification of significant protein quantities to allow for their biochemical characterization and offers a more rapid purification compared to traditional methods, its major limitation is the artificial incorporation of metal ions from the IMAC column. Additionally, these examples highlight the loss of $\mathrm{Fe}^{2+}$ that occurs with the aerobic purification of proteins.

The next logical steps to improve the protein purification process were to find an affinity tag that does not artificially introduce metal ions and to purify the proteins under anaerobic conditions. For these purposes, LpxC from E. coli containing a cleavable (TEV protease) ZZ-tag was purified under anaerobic conditions using IgG-sepharose and elution from the resin by TEV [17]. Purified LpxC expressed in minimal media was shown to contain bound $\mathrm{Fe}(0.4)$ and $\mathrm{Zn}(0.08)$. When the minimal media was supplemented with $\mathrm{Fe}$ and/or $\mathrm{Zn}$ the ratios of bound metal ions shift - Fe supplement: Fe 0.6 and $\mathrm{Zn} \mathrm{0.04;}$ $\mathrm{Zn}$ supplement: $\mathrm{Fe} 0.1$ and $\mathrm{Zn} 0.25$. These results suggest that the LpxC cofactor may switch in response to changing metal environment. The activity of native LpxC was shown to exhibit similar properties to $\mathrm{Fe}^{2+}$ LpxC (i.e., aerobic sensitivity), suggesting that the majority of native EcLpxC is present as $\mathrm{Fe}^{2+}$-LpxC [18]. HDAC8 expressed in minimal media (E. coli) and purified anaerobically using the ZZ-tag was shown to contain bound $\mathrm{Fe}(0.6)$ and $\mathrm{Zn}(0.2)$ [19]. However, unlike LpxC there is no significant change in bound metal content observed upon metal supplementation of minimal media. These results confirm the earlier finding that recombinant HDAC8 expressed in E. coli is predominantly present as $\mathrm{Fe}^{2+}$-HDAC8.

Our attempts to utilize the ZZ-tag for identification of the native cofactors for MshB were unsuccessful due to the low levels of soluble protein expressed using this construct. To circumvent this problem, we have prepared a protein construct containing a TEV cleavable maltose binding protein (MBP)-tag (Kocabas and Hernick, unpublished results) that yielded high levels of soluble MBP-MshB expression. Attempts to purify MshB from amylose resin via TEV cleavage yielded a mixture of MshB, MBP-MshB and MBP, and therefore, could not be used for accurate determination of metal/protein ratios. Additionally, attempts to purify MshB using anti-MBP resin were unsuccessful as TEV could not cleave the MBP-MshB bound to this resin. Consequently, we explored the Halo-tag as a possible affinity tag for native cofactor identification [16]. Halo-MshB was expressed in minimal media and purified anaerobically (TEV-cleavage from Halolink resin) to yield MshB containing bound $\mathrm{Fe}(0.7)$ and $\mathrm{Zn}$ (0.1). Supplementation of the media with $\mathrm{Zn}$ or Fe did not alter the ratios of bound metals when purified under anaerobic conditions, similar to what was observed above with HDAC8. However, aerobic purification of MshB from $E$. coli expressed in media supplemented $\mathrm{Zn}$ or LB results in an enzyme containing a bound $\mathrm{Zn}$ ion (no Fe). The Halo-tag was successful in improving the expression of soluble protein and allows for rapid isolation of protein in single step purification under anaerobic conditions without artificial introduction of metal ions, making it ideal for native cofactor identification studies.

In conclusion, recombinant protein expression and the use of affinity tags have proven to be invaluable resources for the identification and characterization of native cofactors. This technology has enabled the isolation of large quantities of proteins, biochemical characterization of proteins, and aided the identification of native cofactors. Recent studies have identified the Halo-tag as an ideal affinity tag for studies aimed at identifying native cofactors as it: increases soluble protein expression, does not artificially introduce metal ions, and allows for one-step purification of proteins under anaerobic conditions.

\section{References}

1. Morrison C (2009) Financings of the Fortnight: Summer Madness, The In Vivo Blog, Elsevier, Inc.

2. Supuran CT (2010) Carbonic anhydrase inhibitors. Bioorg Med Chem Lett 20: 3467-3474.

3. Lia NG, Shib ZH, Tang YP, Duan JA (2009) Selective matrix metalloproteinase inhibitors for cancer. Curr Med Chem 16: 3805-3827.

4. Drag M, Salvesen GS (2010) Emerging principles in protease-based drug discovery. Nat Rev Drug Discov 9: 690-701.

5. Anthony CS, Masuyer G, Sturrock ED, Acharya KR (2012) Structure based drug design of angiotensin-I converting enzyme inhibitors. Curr Med Chem 19 845-855.

6. Hernick M (2013) Metalloenzymes: Native Co-factor or Experimental Artifact? Biochem Anal Biochem 1: e120.

7. Duerre JA, Miller CH (1966) Cleavage of S-ribosyl-L-homocysteine by extracts from Escherichia coli. J Bacteriol 91: 1210-1217.

8. Miller $\mathrm{CH}$, Duerre JA (1968) S-ribosylhomocysteine cleavage enzyme from Escherichia coli. J Biol Chem 243: 92-97.

9. Rajagopalan PT, Datta A, Pei D (1997) Purification, characterization, and inhibition of peptide deformylase from Escherichia coli. Biochemistry 36 13910-13918.

10. Zhu J, Dizin E, Hu X, Wavreille AS, Park J, et al. (2003) S-Ribosylhomocysteinase (LuxS) is a mononuclear iron protein. Biochemistry 42: 4717-4726.

11. Adams JM (1968) On the release of the formyl group from nascent protein. $J$ Mol Biol 33: 571-589.

12. Ravi Rajagopalan PT, Christopher Yu X, Pei D (1997) Peptide deformylase: A new type of mononuclear iron protein. J Am Chem Soc 119: 12418-12419.

13. Rajagopalan PT, Pei D (1998) Oxygen-mediated inactivation of peptide deformylase. J Biol Chem 273: 22305-22310.

14. Nguyen KT, Wu JC, Boylan JA, Gherardini FC, Pei D (2007) Zinc is the meta cofactor of Borrelia burgdorferi peptide deformylase. Arch Biochem Biophys 468: $217-225$.

15. Gantt SL, Gattis SG, Fierke CA (2006) Catalytic activity and inhibition of human histone deacetylase 8 is dependent on the identity of the active site metal ion. Biochemistry 45: 6170-6178.

16. Huang X, Kocabas E, Hernick M (2011) The activity and cofactor preferences of $\quad \mathrm{N}$-acetyl-1-D-myo-inosityl-2-amino-2-deoxy-alpha-D-glucopyranoside deacetylase (MshB) change depending on environmental conditions. J Bio Chem 286: 20275-20282

17. Gattis SG, Hernick M, Fierke CA (2010) Active site metal ion in UDP-3-O((R)-3-hydroxymyristoyl)-N-acetylglucosamine deacetylase (LpxC) switches between $\mathrm{Fe}(\mathrm{II})$ and $\mathrm{Zn}(\mathrm{II})$ depending on cellular conditions. J Biol Chem 285: 33788-33796.

18. Hernick M, Gattis SG, Penner-Hahn JE, Fierke CA (2010) Activation of Escherichia coli UDP-3-O-[(R)-3-hydroxymyristoyl]-N-acetylglucosamine deacetylase by $\mathrm{Fe} 2+$ yields a more efficient enzyme with altered ligand affinity Biochemistry 49: 2246-2255.

19. Gattis SG (2010) Mechanism and metal specificity of zinc-dependent deacetylases Biological Chemistry, University of Michigan, Ann Arbor, MI, 174 